(2) Open Access Full Text Article

REVIEW

\title{
Involved site radiation therapy for the treatment of early-stage Hodgkin lymphoma in adolescents and young adults
}

This article was published in the following Dove Press journal:

Clinical Oncology in Adolescents and Young Adults

16 October 2015

Number of times this article has been viewed

\author{
Carol S Portlock ${ }^{1,2}$ \\ 'Division of Hematologic Oncology, \\ Department of Medicine, Memorial \\ Sloan Kettering Cancer Center, \\ ${ }^{2}$ Weill Cornell Medical College, \\ New York, NY, USA
}

\begin{abstract}
Radiation therapy technology has permitted the development of new treatment planning techniques. Involved field, involved node, and involved site radiotherapy fields are discussed and compared. Indications for and implications of combined modality therapy are examined, particularly as pertinent to the adolescent and young adult population.
\end{abstract}

Keywords: Hodgkin lymphoma, AYA, involved site or involved node radiotherapy

\section{Introduction}

In a review of the past 50 years of Hodgkin lymphoma (HL) discovery, evaluation, management, and therapy, Canellos et al concluded that

$[\ldots]$ despite large-scale clinical trials $[\ldots]$ in early-stage disease, the question of the relative benefits of radiation therapy alone versus known-to-be-effective drug treatments alone has been inadequately addressed, in part because of ethical considerations in withholding established therapies and in part by specialty competition. ${ }^{1}$

Consequently, the modern era has led primarily to combined modality approaches utilizing combination chemotherapy followed by consolidative radiotherapy. This review will examine the newest consolidative radiotherapy strategies, their definitions, and predicted benefits.

In the first randomized clinical trials for early-stage HL, Kaplan and Rosenberg ${ }^{2}$ introduced the concept and definition of involved and extended radiation therapy (RT) fields. Although crude by current standards, the studies did show the value of extended fields with increased curability. Nevertheless, it was the late effects of radiotherapy, with increased second malignancies and cardiovascular complications, which negatively impacted survival and became the Achilles heel of HL therapy. ${ }^{3,4}$

Likewise, nitrogen mustard, Oncovin ${ }^{\circledR}$ or vincristine, procarbazine, and prednisone (MOPP) was an excellent therapy for advanced stage HL with curability not previously seen with single agents, but the late effects of infertility and myelodysplasia/acute leukemia had a similarly negative survival impact. ${ }^{5}$ Adriamycin, bleomycin, vinblastine, dacarbazine (ABVD) became the combination regimen of choice in advanced stage HL because it had fewer late effects while being equally efficacious as MOPP and MOPP-containing regimens. ${ }^{6}$ Thus, after 50 years, it is now routine to utilize ABVD followed by consolidative RT in early-stage classical HL (cHL) in adolescent, adolescent and young adult (AYA), and adult patients. ABVD has supplanted the need for extended radiotherapy fields but has not yet eliminated
Division of Hematologic Oncology Department of Medicine, Lymphom Service, Memorial Sloan Kettering Cancer Center, 1275 York Avenue, New York, NY 10065, USA

$\mathrm{Tel}+$ I 2126398109

Email portlocc@mskcc.org 
the involved field RT (IFRT) consolidation. In pediatric and AYA cHL, other chemotherapy regimens have also been explored, but the radiotherapy concepts and principles are similar. $^{7-9}$

\section{Involved site radiation therapy}

Recently, the International Lymphoma Radiation Oncology Group (ILROG) published guidelines for a new RT imaging/ planning strategy to update and improve consolidative RT. ${ }^{10}$ Rather than involved field, which was based on the site of disease and the original 1960s Ann Arbor lymphoid regions, ILROG introduced involved site RT (ISRT). Girinsky et al ${ }^{11}$ had introduced involved node RT (INRT), which had a more stringent definition, requiring a prechemotherapy positron emission tomography (PET) in the RT treatment position. ILROG recognized that although a stringent definition was ideal, it was not practical. Therefore, ISRT was adopted, incorporating a prechemotherapy PET, but not requiring that this be in the RT treatment position. Moreover, it utilizes a postchemotherapy treatment planning CT. Most importantly, ISRT is a different concept from IFRT. ISRT is defined as prechemotherapy involved nodes/sites, while IFRT is defined on anatomical boundaries including a whole lymphatic region. In most situations, these volumes may be significantly different.

Of interest is that even with the adoption of ISRT by the radiotherapy community, the application of this new technique is not at all uniform. Hoppe and Hoppe ${ }^{12}$ have recently published the results of a survey of 44 expert lymphoma radiation oncologists. Although only $52 \%$ responded, it is illuminating to review the outcome of this questionnaire, as it demonstrates how little has changed with the advent of new technology and treatment planning techniques. Seven case presentations were provided, and there were no right answers. All of the seven cases demonstrate that ISRT includes all involved sites as noted on initial PET imaging. Importantly, postchemotherapy CT or PET imaging does not change the ISRT field. In other words, this field has the same intent as that employed originally by Rosenberg and Kaplan ${ }^{3}$ utilize RT as the curative modality while chemotherapy, although given first, remains adjunctive.

For example, 1) 48-year old male with stage IIEA cHL of right parotid and ipsilateral high neck. The patient received four cycles of ABVD and had a PET complete response. All expert RT respondents chose to treat the cervical nodes, which were positive at initial PET; the only disagreement was whether to radiate the surgically removed site in the parotid. ISRT did not consider that the patient had a complete remission $(\mathrm{CR})$ to the chemotherapy and that this by itself may be curative, even without the ISRT. 2) A 20-year old female with stage IIXB cHL with supraclavicular, bulky mediastinal, and a diaphragmatic lymph node. She received six cycles of ABVD and achieved a CR, except for some residual PET avidity in the superior mediastinum. The question was basically whether to rely on ABVD for potentially curative benefit in those sites that became PET negative. To these RT experts, ISRT meant treatment of all initially PET-involved sites (56\% selected this option) or to treat only the residual PET-avid bulky site (28\% selected this option). A second question had concurrence among all experts, limiting ISRT to the pericardial node only, when the only other option was to irradiate the whole heart.

In other words, ISRT maintains the original intent of IFRT (include all initially involved sites) but reduces the planned radiation volume. This change in treatment planning can reduce radiation exposure to the organs at risk, for example, the heart. However, ISRT does not take into account the significant tumor shrinkage or PET CR achieved with successful chemotherapy. As a result, the ISRT radiation field to the mediastinum remains substantial, even following six cycles of successful chemotherapy and consequent mediastinal mass reduction.

Finally, under ideal circumstances, the radiation and medical oncologist should evaluate each patient prior to any treatment. When the initial plan is that of combined modality, then the radiotherapy will be applied according to the concepts of ISRT. Hodgson et $\mathrm{al}^{8}$ have emphasized that the risks of late toxicity may be significantly different in every single patient, and a strategy of combined modality therapy may be the best for a certain presentation but not ideal for other presentations (eg, a very young girl with mediastinal disease, where chemotherapy alone might a reasonable option). Again, the decision to use or not to use ISRT in certain situations does not depend on the response to chemotherapy, but on a careful plan of the whole treatment strategy (chemotherapy alone vs combined modality), with consideration of the anatomical presentation and other crucial clinical factors such as age. In some situations, ISRT and IFRT fields will be similar, while in the majority of other cases, they will be substantially different.

In pediatrics, the Children's Oncology Group has conducted studies to utilize response-adapted IFRT in intermediate-risk cHL. ${ }^{13}$ An analysis of treatment failures confirmed that initially involved sites are most likely to relapse after chemotherapy (but not exclusively). The authors speculated that consolidative ISRT may be a strategy to address such areas at risk while reducing the organ and tissue radiation risks of IFRT. This will be addressed in future prospective 
clinical trials. Recently, the American College of Radiology has comprehensively reviewed pediatric combined modality therapy of $\mathrm{cHL}$ and provided recent guidelines. ${ }^{14}$

\section{Principles of ISRT}

ILROG published their field and dose guidelines in 2014. ${ }^{10}$ The principles of ISRT were defined in that publication and are summarized here. The concept of ISRT was outlined at that time, including definitions for many parameters utilized in RT planning: gross tumor volume (or visible tumor), clinical target volume, and planning target volume. The authors made a distinction between primary radiotherapy treatment when the clinical target volume should be more generous, as compared to that with RT consolidation when the margin of the radiotherapy volume is adjusted (contoured) for the accuracy of the prechemotherapy treatment volume, but again, not for the postchemotherapy treatment volume. Moreover, it is important to understand that RT planning is performed in three dimensions, and that ideally, the prechemotherapy imaging can be fused with the CT simulation study when available.

The clinical target volume also requires consideration of quality/accuracy of imaging, changes in volume since imaging (but not tumor response), expected patterns of cHL spread, potential subclinical disease at diagnosis, and adjacent organ constraints. Lymph nodes that are $<5 \mathrm{~cm}$ apart can still be encompassed within one treatment portal, thus enlarging the area at risk for tissue or organ radiation damage. Target volume may also be affected by movement in the chest/upper abdomen, resulting in a 1.5-2 cm margin added in the superior-inferior directions.

Radiation dose takes into consideration the histology (cHL vs NLPHL [nodular lymphocyte predominance HL]), the clinical presentation, and whether there is suspected residual disease following successful chemotherapy. The field size is not affected, but dosing may be, for example, 2,000 cGy (centiGray) for favorable cHL and 3,000 cGy for unfavorable (as defined by German criteria). Increased dosing is considered when there is a residual mass after planned successful chemotherapy, raising concern for residual disease.

High-quality RT planning requires the ability to utilize CT and PET imaging with simulation position and immobility. Even with the most sophisticated imaging, many of the techniques require clinical judgment. Choosing the smallest volume requires considerations of anatomy, positioning, and available techniques (such as intensity modulated RT [IMRT] and the use of breath-hold). These clinical decisions are keys to limiting late organ damage even if ISRT fields are antici- pated. Radiation therapists are often concerned about such tight margins, as they may have the theoretical downside of a geographic marginal miss and the consequent concern for lessened tumor control. However, the risk of geographical miss is low with INRT/ISRT and IMRT methods. ${ }^{15-17}$ Finally, there are no firm guidelines for organ toxicity/late effects in RT lymphoma management, but the principles are adapted from solid tumor oncology.

\section{Late effects of RT}

In pediatrics and in young adults, it is particularly important to remember that the use of consolidative radiotherapy requires treatment planning as discussed above, but also understanding that the late effects to normal organs may follow many years thereafter. ${ }^{8}$ It is the total years at risk or cumulative risk incidence that needs attention, and this is hardest to project when new techniques are employed. For example, Mulrooney et $\mathrm{l}^{18}$ have analyzed major cardiac events in adult survivors of childhood cancer and demonstrated that at 25 years of postthoracic RT, the risk of significant coronary artery disease, congestive heart failure, valvular disease, or pericarditis was $<3 \%$, when the mean dose to the heart was $<1,500 \mathrm{cGy}$. These risks increased to $6 \%-10 \%$ when the cardiac dose was $>3,500 \mathrm{cGy}$. Armstrong et $\mathrm{al}^{19}$ have emphasized that, in addition to the known RT parameters, there are modifiable risk factors that may also contribute to the known late cardiac toxicities. For example, hypertension significantly increased cardiac risk/events and was independently associated with the risk of cardiac death.

A prognostic score for heart failure has recently been derived in this same patient population, utilizing the parameters of sex, age at cancer diagnosis, anthracycline, and chest radiotherapy doses. ${ }^{20}$

Likewise, with the development of second malignancies, the organ at risk (there is no increased risk if the organ is not irradiated) must also be taken into consideration, and breast shielding techniques, for example, have significantly reduced this concern in young women. But the dose administered and the years at risk when small volumes are irradiated must also be taken into consideration in such instances when the breast tissue cannot be spared entirely. In some instances, there may even be a consideration of modifying the RT dose or planned field to limit exposure to an organ at risk.

As RT fields are modified with ISRT, it is critically important that the efficacy of postchemotherapy consolidative RT be continually examined. As pointed out by Hodgson et al, ${ }^{8}$ the new fields may not always be substantially smaller than the prior involved field, particularly in the typical bulky mediastinal mass. This is because neither ISRT nor INRT 
reduce the field size with chemotherapy improvement, but rather, closely conform the field to the prechemotherapy treatment imaging.

The potential future cumulative risks of INRT as compared to IFRT and even prior mantle field irradiation (MFRT) have been assessed in many publications. ${ }^{21-24}$ Most recently, Maraldo et $\mathrm{al}^{25}$ have developed and analyzed simulation plans for ten patients $\leq 18$ years with stage II supradiaphragmatic presentations. As expected, INRT resulted in smaller fields and less organ volume at risk. The estimated excess risk of 2,000 cGy INRT was compared to IFRT and MFRT. For lifetime excess risk of lung cancer and breast cancer, INRT resulted in 2.2 and 2.3 mean risk, as compared to IFRT of 3.0 and 4.0, and MFRT of 3.9 and 8.7, respectively. The expected excess cardiac mortality at 25 years fell from 1.5 with MFRT to 1.0 with IFRT and to 0.7 with INRT. This kind of estimation of future risk is simply an estimate and will await the many years required to confirm this analysis.

\section{Decision making in early-stage cHL}

Finally, the most important question is when must consolidative radiotherapy be applied, understanding that in the pediatric and young adult age group, the late effects of treatment may be substantial. PET-adapted interim restaging has made it possible to address this question prospectively. ${ }^{26}$ Two recent studies in young and older adults illustrate the conundrum of decision making in early-stage nonbulky cHL. Raemaekers et a ${ }^{27}$ studied favorable early-stage cHL (median age 31 years, 15-70 years) and prospectively compared $\mathrm{ABVD} \times 3+\mathrm{INRT}$ vs $\mathrm{ABVD} \times 2$ with either consolidation $A B V D \times 2$ when PET negative or if PET positive, 2 BEACOPPesc + INRT. RT in both groups was 3,000 cGy. This study was closed early when there were nine events in the chemotherapy alone group, whereas the combined modality arm had only one event. Progression-free survival at 1 year was $100 \%$ for ABVD + RT and $94.4 \%$ in the ABVD only group $(P=0.026)$. As emphasized by the authors,

It remains difficult $[\ldots]$ to balance the advantages of immediate cure with increased toxicity against a higher relapse risk after less intensive first-line treatment with its consequences for intensive salvage therapy. Thus, the correct treatment strategy is in the eye of the beholder. ${ }^{27}$

The UK RAPID study prospectively treated 602 patients with stage I-IIA cHL. ${ }^{28}$ Four-hundred and twenty six patients became PET negative after three cycles of ABVD (74.6\%). If PET was negative, patients were randomized to no further therapy (211 patients) or to IFRT (209 patients); if PET was positive, patients received one additional cycle of ABVD + IFRT. Four-hundred and twenty patients (median age 34 years, 16-75 years) were randomized, and the 3 -year progression-free survival favored the RT group (94.6\% vs $90.8 \%$ ), although not statistically significant. Overall survival was similar at 3 years, $97.1 \%$ vs 99\%, respectively. The UK RAPID authors concluded that

Although the noninferiority margin was exceeded in this study, the results suggest that radiotherapy can be avoided for patients with negative PET findings. ${ }^{28}$

They also concluded that, in retrospect, the EORTCLYSA H10 trial of Raemaekers et $\mathrm{al}^{27}$ demonstrated similar results:

[...] radiotherapy after initial chemotherapy marginally improves the progression-free survival rate, as compared with chemotherapy alone, but at the expense of exposing to radiation all patients with negative PET findings, most of whom are already cured. ${ }^{27}$

Thus, the role of consolidative RT is now seriously challenged in early-stage favorable cHL, although both of the above studies were limited to ages of 15-16+. In addition, several recent references examine this important question in detail. $., 26,29$

Bulky cHL remains the one presentation for which the recommendation of consolidative RT remains constant. Importantly, however, the IFRT and the ISRT or INRT fields are not that dissimilar, as outlined by Hodgson et al. ${ }^{8}$ This is due to the fact that all of these radiation fields are designed on the prechemotherapy imaging rather than the postchemotherapy result. Recently, Aznar et $a l^{30}$ have examined the value of deep inspiration breath-hold (DIBH), demonstrating that this simple technique can significantly lessen the potential late effects to mediastinal tissues/organs by pulling the mediastinal mass up and away from the heart and lungs. In a series of RT treatment planning, the excess relative risk was estimated for 22 patient examples, comparing free breathing vs DIBH and in virtually all categories, DIBH was significantly superior: myocardial infarction, cancer risks of thyroid/lung/breast, and life years lost. Perhaps, this simple change in radiotherapy technique will result in these predicted benefits, and clinical trials are ongoing.

\section{Conclusion}

RT techniques continue to be improved and refined. The aim to date has simply been to encompass all known disease at diagnosis and to utilize the administered chemotherapy as an 
adjunct to RT, whether IFRT, ISRT, or INRT. For the future, clinical trials will need to examine further the value of ISRT, of DIBH, but more importantly, the role of adjunctive RT: treating only the postchemotherapy volume. This will become even more relevant as new chemotherapy regimens are introduced, utilizing new drugs such as Brentuximab vedotin. ${ }^{31,32}$

\section{Acknowledgment}

This research was funded in part through the National Institutes of Health/National Cancer Institute Cancer Center Support Grant P30 CAoo8748.

\section{Disclosure}

The author reports no conflicts of interest in this work.

\section{References}

1. Canellos GP, Rosenberg SA, Friedberg JW, Lister TA, DeVita VT. Treatment of Hodgkin lymphoma: a 50-year perspective. J Clin Oncol. 2014;32(3):163-168.

2. Kaplan HS, Rosenberg SA. Extended-field radical radiotherapy in advanced Hodgkin's disease: short-term results of 2 randomized clinical trials. Cancer Res. 1966;26(6):1268-1276.

3. Rosenberg SA, Kaplan HS. The evolution and summary results of the Stanford randomized clinical trials of the management of Hodgkin's disease:1962-1984. Int J Radiat Oncol Biol Phys. 1985;11(1):5-22.

4. Donaldson SS, Glatstein E, Rosenberg SA, Kaplan HS. Pediatric Hodgkin's disease. II. Results of therapy. Cancer. 1976;37(5):2436-2447.

5. Coleman CN, Williams CJ, Flint A, Glatstein EJ, Rosenberg SA, Kaplan HS. Hematologic neoplasia in patients treated for Hodgkin's disease. N Engl J Med. 1977;297(23):1249-1252.

6. Duggan DB, Petroni GR, Johnson JL, et al. Randomized comparison of ABVD and MOPP/ABV hybrid for the treatment of advanced Hodgkin's disease: report of an intergroup trial. J Clin Oncol. 2003; 21(4):607-614.

7. Giulino-Roth L, Keller FG, Hodgson DC, Kelly KM. Current approaches in the management of low risk Hodgkin lymphoma in children and adolescents. Br J Haematol. 2015;169(5):647-660.

8. Hodgson DC, Dieckmann K, Terezakis S, Constine L; International Lymphoma Radiation Oncology Group. Implementation of contemporary radiation therapy planning concepts for pediatric Hodgkin lymphoma: guidelines from the International Lymphoma Radiation Oncology Group. Pract Radiat Oncol. 2015;5(2):85-92.

9. Crump M, Herst J, Baldassarre F, et al. Evidence-based focused review of the role of radiation therapy in the treatment of early-stage Hodgkin lymphoma. Blood. 2015;125(11):1708-1716.

10. Specht L, Yahalom J, Illidge T, et al; ILROG. Modern radiation therapy for Hodgkin lymphoma: field and dose guidelines from the International Lymphoma Radiation Oncology Group (ILROG). Int J Radiat Oncol Biol Phys. 2014;89(4):854-862.

11. Girinsky T, van der Maazen R, Specht L, et al. Involved-node radiotherapy (INRT) in patients with early Hodgkin lymphoma: concepts and guidelines. Radiother Oncol. 2006;79(3):270-277.

12. Hoppe BS, Hoppe RT. Expert radiation oncologist interpretations of involved-site radiation therapy guidelines in the management of Hodgkin lymphoma. Int J Radiat Oncol Biol Phys. 2015;92(1):40-45.

13. Dharmarajan KV, Friedman DL, Schwartz CL, et al. Patterns of relapse from a phase 3 Study of response-based therapy for intermediate-risk Hodgkin lymphoma (AHOD0031): a report from the Children's Oncology Group. Int J Radiat Oncol Biol Phys. 2015;92(1):60-66.

14. Terezakis SA, Metzger ML, Hodgson DC, et al. ACR Appropriateness Criteria Pediatric Hodgkin Lymphoma. Pediatr Blood Cancer. 2014; 61(7):1305-1312.
15. Campbell BA, Voss N, Pickles T, et al. Involved-nodal radiation therapy as a component of combination therapy for limited-stage Hodgkin's lymphoma: a question of field size. J Clin Oncol. 2008;26(32):5170-5174.

16. Maraldo MV, Aznar MC, Vogelius IR, Petersen PM, Specht L. Involved node radiation therapy: an effective alternative in early-stage hodgkin lymphoma. Int J Radiat Oncol Biol Phys. 2013;85(4):1057-1065.

17. Filippi AR, Ciammella P, Piva C, et al. Involved-site image-guided intensity modulated versus 3D conformal radiation therapy in early stage supradiaphragmatic Hodgkin lymphoma. Int J Radiat Oncol Biol Phys. 2014;89(2):370-375.

18. Mulrooney DA, Yeazel MW, Kawashima T, et al. Cardiac outcomes in a cohort of adult survivors of childhood and adolescent cancer: retrospective analysis of the Childhood Cancer Survivor Study cohort. BMJ. 2009;339:b4606.

19. Armstrong GT, Oeffinger KC, Chen Y, et al. Modifiable risk factors and major cardiac events among adult survivors of childhood cancer. J Clin Oncol. 2013;31(29):3673-3680.

20. Chow EJ, Chen Y, Kremer LC, et al. Individual prediction of heart failure among childhood cancer survivors. J Clin Oncol. 2015;33(5): 394-402.

21. Hodgson DC, Koh ES, Tran TH, et al. Individualized estimates of second cancer risks after contemporary radiation therapy for Hodgkin lymphoma. Cancer. 2007;110(11):2576-2586.

22. Filippi AR, Ragona R, Fusella M, Botticella A, Fiandra C, Ricardi U. Changes in breast cancer risk associated with different volumes, doses, and techniques in female Hodgkin lymphoma patients treated with supra-diaphragmatic radiation therapy. Pract Radiat Oncol. 2013;3(3): 216-222.

23. Weber DC, Johanson S, Peguret N, Cozzi L, Olsen DR. Predicted risk of radiation-induced cancers after involved field and involved node radiotherapy with or without intensity modulation for early-stage hodgkin lymphoma in female patients. Int J Radiat Oncol Biol Phys. 2011;81(2):490-497.

24. Koh ES, Tran TH, Heydarian M, et al. A comparison of mantle versus involved-field radiotherapy for Hodgkin's lymphoma: reduction in normal tissue dose and second cancer risk. Radiat Oncol. 2007;2:13.

25. Maraldo MV, Jørgensen M, Brodin NP, et al. The impact of involved node, involved field and mantle field radiotherapy on estimated radiation doses and risk of late effects for pediatric patients with Hodgkin lymphoma. Pediatr Blood Cancer. 2014;61(4):717-722.

26. Sickinger MT, von Tresckow B, Kobe C, Engert A, Borchmann P, Skoetz N. Positron emission tomography-adapted therapy for first-line treatment in individuals with Hodgkin lymphoma. Cochrane Database Syst Rev. 2015;1:CD010533.

27. Raemaekers JM, André MP, Federico M, et al. Omitting radiotherapy in early positron emission tomography-negative stage I/II Hodgkin lymphoma is associated with an increased risk of early relapse: clinical results of the preplanned interim analysis of the randomized EORTC/ LYSA/FIL H10 trial. J Clin Oncol. 2014;32(12):1188-1194.

28. Radford J, Illidge T, Counsell N, et al. Results of a trial of PETdirected therapy for early-stage Hodgkin's lymphoma. $N$ Engl J Med. 2015;372(17):1598-1607.

29. Hay AE, Klimm B, Chen BE, et al; Conducted by the NCIC Clinical Trials Group (Canada) and German Hodgkin Study Group (GHSG). An individual patient-data comparison of combined modality therapy and ABVD alone for patients with limited-stage Hodgkin lymphoma. Ann Oncol. 2013;24(12):3065-3069.

30. Aznar MC, Maraldo MV, Schut DA, et al. Minimizing late effects for patients with mediastinal Hodgkin lymphoma: deep inspiration breath-hold, IMRT, or both? Int J Radiat Oncol Biol Phys. 2015;92(1): 169-174.

31. Stathis A, Younes A. The new therapeutical scenario of Hodgkin lymphoma. Ann Oncol. 2015;00:1-8.

32. Kumar A, Yahalom J, Schoder H, et al. Safety and early efficacy in an ongoing pilot study of brentuximab vedotin and AVD chemotherapy followed by 30 gray involved-site radiotherapy for newly diagnosed, early stage, unfavorable risk hodgkin lymphoma. Blood. 2014;124:3085. 


\section{Publish your work in this journal}

Clinical Oncology in Adolescents and Young Adults is an international, peer-reviewed, open access journal publishing original research, reports, editorials, reviews and commentaries on all aspects of epidemiology, diagnosis and treatment of cancers in adolescents and young adults. The manuscript management system is completely online and includes a very quick and fair peer-review system. Visit http://www.dovepress.com/testimonials.php to read real quotes from published authors.

\footnotetext{
Submit your manuscript here: http://www.dovepress.com/clinical-oncology-in-adolescents-and-young-adults-journal
} 